Revue

Revue de l'histoire des religions

de Ihistoire des religions

$4 \mid 2012$

Varia

\title{
Anne-Claire HUSSER, Bruno BARTHELMÉ, Nicolas PIQUÉ (dir.), Les sources de la morale laïque : héritages croisés
} Lyon, ENS Éditions, 2009, 130 p., 22 cm, 15 €, ISBN 978-2-84788-199-8.

Éric Dubreucq

\section{CpenEdition}

Journals

Édition électronique

URL : http://journals.openedition.org/rhr/8015

DOI : $10.4000 /$ rhr.8015

ISSN : 2105-2573

Éditeur

Armand Colin

Édition imprimée

Date de publication : 1 décembre 2012

Pagination : $560-562$

ISBN : 978-2200-92796-7

ISSN : 0035-1423

Référence électronique

Éric Dubreucq, « Anne-Claire husser, Bruno barthelmé, Nicolas piaué (dir.), Les sources de la morale lä̈que héritages croisés », Revue de l'histoire des religions [En ligne], 4 | 2012, mis en ligne le 24 janvier 2013, consulté le 22 septembre 2020. URL : http://journals.openedition.org/rhr/8015 ; DOI : https://doi.org/ $10.4000 /$ rhr.8015

Ce document a été généré automatiquement le 22 septembre 2020.

Tous droits réservés 


\section{Anne-Claire HUSSER, Bruno BARTHELMÉ, Nicolas PIQUÉ (dir.), Les sources de la morale laïque : héritages croisés}

Lyon, ENS Éditions, 2009, 130 p., 22 cm, 15 €, ISBN 978-2-84788-199-8.

\section{Éric Dubreucq}

\section{RÉFÉRENCE}

Anne-Claire HUSSER, Bruno BARTHELMÉ, Nicolas PIQUÉ (dir.), Les sources de la morale läque : héritages croisés, Lyon, ENS Éditions, 2009, 130 p., 22 cm, 15 €, ISBN 978-2-84788-199-8.

1 Ce volume, qui propose les actes d'une journée de travail organisée en novembre 2007 à l'ENS de Lyon, comporte deux parties consacrées, l'une, à l'étude de « la morale laïque » et de la "pédagogie républicaine ", l'autre, à l'interrogation sur la place de « l'héritage protestant " dans l'instauration de la laïcité, et les rapports de celle-ci avec la « Réforme religieuse ».

2 Les études de la première partie proposent de modifier le regard habituellement porté sur les sources de la pensée éducative et de la morale des intellectuels, fondateurs en France de la laïcité dans le dernier quart du XIX ${ }^{e}$ siècle, en même temps que des valeurs propagées par l'école et par l'éducation publique à cette époque. Dans le chapitre consacré à « l'école de Jules Ferry : une laïcité positiviste? », Pierre Kahn interroge et met à mal la thèse jadis défendue par Louis Legrand et Claude Nicolet, en exhumant, par une lecture précise et attentive des écrits de Ferry (en particulier dans le discours sur l'égalité d'éducation d'avril 1870 et la lettre circulaire aux instituteurs de 1883), la présence de thèmes libéraux, qu'il rapproche des analyses tocquevilliennes de la démocratie en Amérique. Ce chapitre nuancé, au terme duquel l'auteur conclut que «l'enseignement laïque de la morale » ne peut être " qualifié de positiviste », mais peut 
cependant «être compris par référence au positivisme» (p.60), converge avec l'examen mené par Bruno Barthelmé de «l'influence de la philosophie des Lumières sur la formation de la morale laïque ». Dans les deux cas, il s'agit de souligner l'impossibilité de réduire la laïcité aux thèses d'un seul auteur, fût-il Jules Ferry, et même, de réduire les idées d'un auteur à une seule source univoque et singulière. Contre les critiques du livre de Claude Lelièvre et Christian Nique (La République n'éduquera plus. La fin du mythe Ferry, Paris, Plon, 1993), qui tentait de démontrer que l'école de Jules Ferry, en instaurant une éducation morale et civique, avait trahi l'idéal condorcétien d'une instruction refusant toute inculcation de valeurs d'État, l'intention est ici d'examiner finement la manière dont certains thèmes de la philosophie des Lumières sont tantôt repris, tantôt critiqués durant cette période. Ces analyses montrent que l'opposition d'une éducation, entendue comme système d'inculcation de valeurs et de principes opérée par un "État-nation-éducateur ", avec une instruction, c'est-à-dire une purification de l'éducation réduite à la simple culture de la raison et des lumières d'un jugement qui se cantonnerait au fait, est sans doute une fausse manière de poser les problèmes. La diversité des influences présidant à l'instauration de la manière dont le "modèle éducatif républicain » a voulu "moraliser l'enfance populaire ", que souligne Patrick Dubois, converge vers les mêmes conclusions. «La » morale laïque suppose en réalité une diversité d'origines, les unes liées aux philosophies spiritualistes, les autres à la théorie buissonienne de l'intuition, d'autres encore à des conceptions de l'obligation et de la civilisation, dont le Dictionnaire de pédagogie et d'instruction primaire conserve la marque. Là encore, la diversité et la complexité président à la mise en ordre de la doctrine laïque.

3 La seconde partie de l'ouvrage s'attache à dévoiler la même diversité dans les sources de la laïcité et dans les questions religieuses que son instauration affronte. Laurence Loeffel dévoile ainsi une « construction spiritualiste de la morale laïque », dans laquelle l'influence des élèves de Cousin - Paul Janet, Jules Simon, Jules Barni - et de certains de ses héritiers - Elme Caro, Adolphe Franck, ou Henri Marion - a joué un rôle important. De même, dans les pages qu'il consacre à « la question du signe chez Luther, Calvin et Pascal », Nicolas Piqué propose des «éléments pour une généalogie de la laïcité » dont l'intention serait non pas d'en déterminer une origine, autour de l'engagement pour ou contre la République, mais d'en retracer la «longue histoire, relevant à la fois de la morale et de la religion» (p. 79), en montrant par exemple comment les querelles théologiques qui se sont déroulées autour des signes, et la manière d'y interroger les rapports entre le monde profane et visible, monde politique et social des hommes, et le lieu invisible du divin et des vérités absolues, ont constitué le lieu d'élaboration d'une matrice de pensée dans laquelle les philosophies morales et politiques laïques ont pris naissance. La complexité des relations d'engendrement, de différenciation et d'opposition entre la laïcité et sa morale, d'une part, et des strates plus anciennes d'ordre religieux, paraît de manière concrète, enfin, dans le travail proposé par AnneClaire Husser sur «le Sébastien Castellion de Ferdinand Buisson ». Le but est alors de montrer comment les deux questions de la tolérance religieuse et de la morale laïque se différencient, mais aussi comment les conceptions de la tolérance, étudiées par Buisson sur le cas de Castellion, ont fourni l'occasion du développement d'un « individualisme théologique » qui, pour mieux sauvegarder le principe premier de la liberté absolue de la conscience, est amené à reléguer au second rang l'exigence d'unité du dogme et de pouvoir des instituions religieuses et des Églises. Il est alors éclairant de retrouver, dans la conception que Buisson échafaude de la laïcité, le même principe d'une liberté 
individuelle qui, pour se déployer, ne doit être bridée par aucune autorité d'imposition dogmatique, et se donne alors pour un élan du sujet conscient cherchant à progresser vers la réalisation d'idéaux. "L'individualisme moral et politique de Buisson», qui marque en profondeur le système de la pensée laïque en France, «fait ainsi pendant à son individualisme théologique » qui fournit à la laïcité sa «matrice » (p. 100). La leçon de ces études - sur laquelle une réflexion actuelle pourrait, de manière fructueuse, se fonder - paraît être que la laïcité consiste en un système à la fois complexe et multiple, voire hétérogène, dont la force n'est pas tant de former une doctrine une et unique, mais de permettre le rassemblement et la synthèse d'un ensemble pluriel : les hommes et les idées laïques. Cette leçon du passé pourrait valoir pour notre temps présent.

\section{AUTEURS}

\section{ÉRIC DUBREUCQ}

IUFM de l'Université de Franche-Comté. 